\title{
Erratum: Obtaining self-similar scalings in focusing flows [Phys. Rev. E 92, 043016 (2015)]
}

\author{
Joshua A. Dijksman ${ }^{\circledR}$, Shomeek Mukhopadhyay, Cameron Gaebler, Thomas P. Witelski, and Robert P. Behringer
}

(Received 7 May 2020; published 22 May 2020)

DOI: 10.1103/PhysRevE.101.059902

The paper has three small errors that warrant the publication of an erratum to avoid confusion. Conclusions are not affected. The errors are as follows:

(1) The abstract mentions the work "Diez et al. [Q. Appl. Math. 210, 155 (1990)] and the reference is further cited as Ref. [12]. This reference is not correct; it should be J. A. Diez, J. Gratton, and F. Minotti, Self-similar solutions of the second kind of nonlinear diffusion-type equations, Quart. Appl. Math. 50, 401 (1992).

(2) Equation (2) is incorrectly stated. This equation should be the same as Eq. (4.6) from Ref. [20], but it is not. The correct equation was used to generate Fig. 1(f) from the paper; we checked this and can reproduce the plot. The correct equation should be as follows:

$$
R_{S}=R\left[1-\frac{1}{R}\left(\frac{4 g H_{0}}{\Omega^{2}}\right)^{1 / 2}\right]^{1 / 2} .
$$

(3) The critical rotation rate (defined at the beginning of Sec. II A on p. 3) $\Omega_{c}$ was given as $\sqrt{\frac{g R^{2}}{2 H_{0}}}$. This is not correct. The correct critical rotation rate is $\Omega_{c}=\frac{2 \sqrt{g H_{0}}}{R}$ coming from the corrected Eq. (2) mentioned above. 